\title{
Industry updates from the field of stem cell research and regenerative medicine in July 2019
}

\author{
Dusko Ilic*,1(iD), Mirjana Liovic ${ }^{2} \&$ Laila Noli \\ ${ }^{1}$ Stem Cell Laboratories, Guy's Assisted Conception Unit, Department of Women \& Children's Health, Faculty of Life Sciences \& \\ Medicine, King's College London, London, UK \\ ${ }^{2}$ Medical Center for Molecular Biology, Faculty of Medicine, University of Ljubljana, Ljubljana, Slovenia \\ ${ }^{3}$ Department of Pathological Sciences, Fakeeh College for Medical Sciences, Jeddah, Kingdom of Saudi Arabia \\ *Author for correspondence: dusko.ilic@kcl.ac.uk
}

Latest developments in the field of stem cell research and regenerative medicine compiled from publicly available information and press releases from nonacademic institutions in July 2019.

First draft submitted: 8 August 2019; Accepted for publication: 8 August 2019; Published online: 29 August 2019

Keywords: industry $\bullet$ regenerative medicine $\bullet$ stem cell

\section{Business development \\ Collaborations, partnerships \& alliances \\ Alliance agreement: ElevateBio \& $M G H$}

ElevateBio (MA, USA; www.elevate.bio), a cell and gene therapy holding company, and Massachusetts General Hospital (MGH; MA, USA; www.massgeneral.org) have entered into a 10-year alliance agreement [1]. The agreement provides MGH-preferred access to ElevateBio's BaseCamp research, process development and manufacturing facility in MA, USA for development and production of highly innovative cell and gene therapies developed at MGH. Under this agreement, MGH is making an investment in ElevateBio BaseCamp, and BaseCamp's facilities and expert staff will support a range of cell- and gene-therapy programs arising from MGH's research programs and laboratories. In addition, ElevateBio and MGH will jointly identify innovative cell and gene technologies from university labs and other external sources to create therapeutics companies to advance additional cell and gene therapies from the lab to the bedside of patients suffering from severe diseases. According to the terms of the agreement, MGH will have guaranteed access to ElevateBio's BaseCamp for multiple, simultaneous cell- and gene-therapy programs for process development and manufacturing each year. At the same time, ElevateBio and MGH may jointly form any number of companies to manufacture and develop cell- and gene-therapeutic candidates from multiple sources.

\section{Collaboration agreement: FUJIFILM \& Axcelead}

FUJIFILM Corporation (Japan; www.fujifilm.com) and Axcelead Drug Discovery Partners (Japan; www.axcele ad.com/en/) have announced a collaboration to provide customers with a human-induced pluripotent stem cell (iPSC)-based integrated platform for drug discovery solutions [2]. By combining Fujifilm's iPSC-derived products that its US subsidiary FUJIFILM Cellular Dynamics (WI, USA; https://fujifilmcdi.com) offers and Axcelead's compound evaluation and analytical services, the companies will work toward developing new drug.

\section{Licensing agreement: Epistem \& HUB}

Epistem (UK; www.epistem.co.uk), a preclinical and clinical research services company, has announced the expansion of its in vitro model portfolio by the completion of an agreement with Hubrecht Organoid Technology (HUB; The Netherlands; www.hub4organoids.eu) to enable the delivery of an increased range of multi species organoid models [3]. Services are focused on the identification of off target or off tissue intestinal toxicities associated 
with various therapeutics. Specifically, Epistem aims to help customers define whether there are reasons to suspect that there may be human toxicity issues. This will allow better prioritization of lead compounds, with customers able to make early decisions on whether to abandon, identify alternatives or develop mitigation strategies for any drugs with human toxicity.

\section{Licensing agreement: Frequency \& Astellas}

Frequency Therapeutics (MA, USA; www.frequencytx.com) and Astellas Pharma (Japan; www.astellas.com/en) have entered into an exclusive license agreement to develop and commercialize Frequency's regenerative therapeutic candidate, FX-322, for the treatment of sensorineural hearing loss, the most common type of hearing loss [4]. At present, there are no approved therapeutic options for sensorineural hearing loss. Under the terms of the agreement, Astellas will be responsible for the development and commercialization of FX-322 outside of the USA and Frequency will be responsible for US development and commercialization. The companies will be jointly responsible for conducting global clinical studies and coordinating commercial launch activities. Frequency will receive an upfront payment of US $\$ 80$ million and may also receive up to an additional US $\$ 545$ million based on development and commercial milestones, as well as royalties on any future product sales in the licensed territory.

Frequency recently completed a Phase I/II clinical study in the USA in which FX-322 was observed to be well tolerated following a single intratympanic injection, with no serious adverse events. Improvements in hearing function were observed in multiple FX-322-treated patients (https://clinicaltrials.gov; ID: NCT03300687). Frequency plans to initiate a Phase IIa study in the fourth quarter of 2019.

\section{Licensing agreement: Humanigen \& Mayo Clinic}

Humanigen (CA, USA; www.humanigen.com) has entered into an exclusive worldwide license with the Mayo Clinic to create CAR-T cells lacking granulocyte-macrophage colony-stimulating factor (GM-CSF), also known as colony-stimulating factor 2, expression through various gene-editing tools including CRISPR-Cas9 [5]. The license covers various patent applications and know-how developed by Mayo in collaboration with Humanigen. These licensed technologies complement and broaden Humanigen's leadership position in the GM-CSF neutralization space and expand Humanigen's discovery platform aimed at improving CAR-T to include gene-edited CAR-T cells.

Acute toxicities such as neurotoxicity and cytokine release syndrome remain a critical unmet need in CAR-T therapy. These toxicities necessitate inpatient therapy, drive unfavorable inpatient reimbursement, are associated with an overall $15 \%$ nondisease relapse mortality rate 1 , and increase the overall costs of CAR-T therapy. Strategies to improve the safety profile of CAR-T without negatively impacting efficacy are needed to improve its benefit-risk profile, cost-effectiveness and to enable CAR-T to move beyond use solely in relapsed/refractory patients to earlier lines of therapy.

A strong link exists between efficacy and toxicity of current and next generation CAR-T therapies, including allogeneic CAR-T, suggesting that neurotoxicity and cytokine release syndrome might be on-target effects. However, clinical correlative analysis and preclinical modeling evidence points to GM-CSF and myeloid cells as key to the inflammatory cascade. Emerging evidence suggests that GM-CSF is the communication conduit between the ontarget CAR-T/tumor engagement and the nonspecific and off-target inflammatory cascade caused by myeloid cells resulting in neurotoxicity and cytokine release syndrome. GM-CSF neutralization is emerging as a potential key switch to shutting down the off-target inflammatory cascade while preserving the on-target efficacy of the CAR$\mathrm{T}$ therapy, thereby breaking the efficacy/toxicity linkage. With this license agreement, Humanigen significantly expands its intellectual property portfolio to include gene-edited CAR-T cells which can be engineered to lack the ability of producing GM-CSF, which may improve the efficacy and safety profile of CAR-T.

\section{Licensing agreement: Humanigen \& University of Zurich}

Humanigen has secured an exclusive worldwide license agreement from the University of Zurich (UZH; Switzerland; www.uzh.ch/en.html) for technology used to prevent graft-versus-host disease through GM-CSF neutralization [6]. The license covers various patent applications filed by UZH which complement and broaden Humanigen's leadership position in the application of GM-CSF and expand Humanigen's development platform to include improving allogeneic hematopoietic stem cell transplantation. 


\section{Launching new products, services \\ Anemocyte}

In collaboration with RPS Aerospace (Italy; www.rpsaerospace.com/en/), Anemocyte (Italy; www.anemocyte.co m) developed a remotely piloted drone system to securely transport final cell and gene therapy products from manufacturing sites to clinical centers [7]. The current prototype can also transport apheresis material, bone marrow and biopsy specimens and is equipped with GPS tracking, antitampering technology, temperature control and continuous real-time monitoring of these parameters during flights. At this time, all applications are for research and development purposes only, and the first simulation flight, a 50-km route, is anticipated for later this year.

\section{Kite}

Kite (CA, USA; www.kitepharma.com), a Gilead Company (CA, USA; www.gilead.com) has announced plans for a new 67,000- $\mathrm{ft}^{2}$ facility in CA, USA dedicated to the development and manufacturing of viral vectors, a critical starting material in the production of cell therapies [8]. The new site builds on Kite's existing state-ofthe-art manufacturing capabilities to deliver innovative cell therapies for people with cancer, including Yescarta (axicabtagene ciloleucel), Kite's first commercially available chimeric antigen receptor T (CAR T) cell therapy, and investigational T-cell receptor and tumor neoantigen targeting cell therapies being evaluated in solid tumors.

\section{StemoniX}

Stemoni ${ }^{\circledR}$ (CA, USA; www.stemonix.com) has opened its new, state-of-the-art research \& development facility in CA, USA [9]. The facility will house StemoniX's research team, who will concentrate on developing new preclinical applications, disease modeling techniques and drug discovery opportunities involving the company's high-throughput microOrgan technology platforms. microOrgans are the world's first ready-to-use assay plates containing living microtissues engineered from human-iPSCs (hiPSCs). Designed to enable high-throughput human drug screening without the need for drugs to enter a human body, microOrgans, including microBrain ${ }^{\circledR}$ and microHeart ${ }^{\circledR}$, offer the potential to deliver significant cost and time savings to the pharmaceutical industry by enhancing the safety, consistency, and accuracy of human clinical trials that today rely on animal models and limited human testing as predictors of drug interactions across an entire patient population.

\section{Clinical trials}

\section{Pluripotent stem cells}

BioTime

BioTime (CA, USA; www.biotimeinc.com) has announced that it has dosed its first patient with the Orbit Subretinal Delivery System as well as with a new Thaw-and-Inject formulation of OpRegen ${ }^{\circledR}$, the Company's retinal pigment epithelium (RPE) transplant therapy, in its ongoing Phase I/IIa clinical study for the treatment of dry age-related macular degeneration, a leading cause of adult blindness in the developed world [10]. OpRegen consists of a suspension of RPE cells delivered subretinally as an intraocular injection. RPE cells are essential components of the back lining of the retina and function to help nourish the retina including photoreceptors. OpRegen has been granted Fast Track designation from the US FDA. OpRegen is a registered trademark of Cell Cure Neurosciences (CA, USA; www.cellcureneurosciences.com), a majority-owned subsidiary of BioTime.

This is a Phase I/IIa open-label, dose escalation safety and efficacy study of human embryonic stem cell-derived RPE cells transplanted subretinally in patients with advanced dry age-related macular degeneration with geographic atrophy. The study will enroll approximately 24 subjects, divided into four cohorts. The first two cohorts, each consisting of three legally blind subjects with best corrected visual acuity (BCVA) of 20/200 or less, received a single subretinal implantation of OpRegen. The third cohort included six subjects with BCVA of 20/200 or less, who received a single subretinal implantation of OpRegen. Staggered intervals within and between cohorts are applied to ensure subject safety and welfare. The fourth cohort will include approximately 12 subjects with BCVA between 20/64 and 20/250, who will receive a single subretinal implantation of OpRegen. Cohort 4 includes two formulations of OpRegen; the first three subjects were treated with an ophthalmic Balanced Salt Solution Plus version of OpRegen. Remaining subjects will be treated with an 'off-the-shelf' or 'thaw and inject' formulation. OpRegen thaw and inject can be shipped directly to sites and used upon thawing, which removes the complications and logistics of having to use a dose preparation facility. Staggered intervals will be applied between at least the first two subjects of each delivery modality to ensure subject safety and welfare. 
T cells

Adaptimmune

Adaptimmune Therapeutics (UK, www.adaptimmune.com), a leader in T-cell therapy to treat cancer, has initiated SURPASS, its first clinical trial with a next-generation Specific Peptide Enhanced Affinity Receptor T (SPEAR-T) cell targeting MAGE-A4 (https://clinicaltrials.gov; ID: NCT04044859) [11]. This next-generation SPEAR T-cell, known as ADP-A2M4CD8, expresses the CD8 $\alpha$ coreceptor alongside the engineered T-cell receptor that targets MAGE-A4. Preclinical data indicate that co-expression of CD8 $\alpha$ may broaden the immune response against solid tumors and increase antitumor activity by leveraging CD4 cells into CD8 killer or cytotoxic T cells while retaining their $\mathrm{CD}^{+}$helper function. The SURPASS trial will enroll up to 30 patients across multiple solid tumor indications. Like Adaptimmune's ongoing trials, the SURPASS trial will be a three-cohort dose escalation study. Unlike the other trials, the stagger between patients will be shorter and the starting dose in the first cohort will be 0.8-1.2 billion SPEAR T cells, instead of 100 million SPEAR T-cells. Each dose cohort will enroll three patients and can be expanded to six patients if a dose limiting toxicity occurs. The dose ranges for the other two cohorts are: 1.2-3 billion and 3-6 billion SPEAR T cells. After dose escalation is complete, there is an expansion phase with doses up to 10 billion cells.

\section{Adaptimmune}

Adaptimmune Therapeutics has started its SPEARHEAD-1 trial with ADP-A2M4 SPEAR T cells for patients with synovial sarcoma or myxoid/round cell liposarcoma (https://clinicaltrials.gov; ID: NCT04044768) [12]. Earlier this year, Adaptimmune presented compelling data with responses observed in synovial sarcoma patients treated in the ADP-A2M4 pilot study. The SPEARHEAD-1 trial will treat up to 60 patients with inoperable or metastatic synovial sarcoma or myxoid/round cell liposarcoma who have received prior chemotherapy. Patients will receive doses of up to 10 billion ADP-A2M4 SPEAR T cells and the lymphodepletion regimen of fludarabine $\left(30 \mathrm{mg} / \mathrm{m}^{2} /\right.$ day for 4 days) and cyclophosphamide (600 mg/ $\mathrm{m}^{2} /$ day for 3 days). The primary end point is overall response rate by RECIST v1.1 by independent review. Safety endpoints will be reviewed by an Independent Data Safety Monitoring Board (DSMB).

\section{Other}

Elios

Elios Therapeutics (TX, USA; www.eliostherapeutics.com), a biopharmaceutical company developing innovative autologous, particle-delivered, dendritic cell cancer vaccines, has announced positive top-line results from the company's prospective, randomized, double-blind, placebo-controlled Phase IIb clinical trial evaluating its lead immuno-oncology candidate, the TLPLDC (tumor lysate, particle-loaded, dendritic cell) vaccine, in patients with Stage III and IV resected melanoma (https://clinicaltrials.gov; ID: NCT02678741) [13]. The study met its primary end point by demonstrating a statistically significant reduction in the risk of disease recurrence at 24-months (disease-free survival; [DFS]) in the per treatment (PT) population.

The prespecified primary efficacy analysis was performed on the intent-to-treat and the PT populations as coprimary analyses given the high early recurrence rate often seen in patients with high-risk melanoma and the time needed for vaccines to activate the immune system. For all 144 patients enrolled in the study, including those who were never, or incompletely vaccinated, the recurrence rate was $66 \%$ in the placebo arm compared with $54 \%$ in the vaccine arm, representing an $18 \%$ clinically meaningful, though statistically nonsignificant, reduction in the relative risk of disease recurrence. However, the PT analysis of all patients who completed the primary vaccine series (6 months) of TLPLDC or placebo, demonstrated a 56\% recurrence rate in the placebo arm versus $29 \%$ in the vaccine arm, representing a highly statistically significant $50 \%$ reduction in the relative risk of disease recurrence.

The independent DSMB responsible for evaluating the results of the study determined that there were no safety concerns with only one-third of patients experiencing a related adverse event, the majority of which were grade 1 or 2. Furthermore, an initial assessment of 36-month follow-up data on all patients indicates that the TLPLDC vaccine benefit is not only durable, but continues to show benefit beyond 24 months. As a result, the DSMB recommended that the study continue as designed to the 36-month landmark end points of DFS and overall survival, anticipated in June 2020. 


\section{Regulations, approvals, acquisitions... Green light \\ Caladrius}

Caladrius Biosciences (NJ, USA; www.caladrius.com) has announced that the European Medicines Agency has granted Advanced Therapy Medicinal Product classification to the CLBS12, autologous G-CSF-mobilized peripheral blood-derived CD34+ ${ }^{+}$cell product, for the treatment of critical limb ischemia [14]. CLBS12 has previously been awarded a SAKIGAKE designation in Japan, a status which makes the product eligible for early conditional approval based on the on-going clinical trial in that country.

\section{Mustang Bio}

Mustang Bio (NY, USA; www.mustangbio.com) has announced that the FDA has granted Orphan Drug Designation to MB-102 (CD123 CAR T) for the treatment of acute myeloid leukemia (AML) [15]. The FDA also previously granted Orphan Drug Designation to MB-102 (CD123 CAR T) for the treatment of blastic plasmacytoid dendritic cell neoplasm (BPDCN). MB-102 (CD123 CAR T) is a CAR T-cell therapy that is produced by engineering patient $\mathrm{T}$ cells to recognize and eliminate CD123-expressing tumors. CD123 is widely expressed on bone marrow cells of patients with myelodysplastic syndromes, as well as in hematologic malignancies, including AML, B-cell acute lymphoblastic leukemia, hairy cell leukemia, BPDCN, chronic myeloid leukemia and Hodgkin's lymphoma. MB-102 is currently being studied in a City of Hope, first-in-human Phase I dose-escalation clinical trial evaluating the safety and antitumor activity of escalating doses of MB-102 in patients with relapsed or refractory AML (cohort 1) and BPDCN (cohort 2) (https://clinicaltrials.gov; ID: NCT02159495). Patients receive a single dose of MB-102 with an option for a second infusion if they continue to meet safety and eligibility criteria and still have CD123 ${ }^{+}$ disease. MB-102 has demonstrated complete responses at low doses in AML and BPDCN without dose-limiting toxicities. City of Hope developed CD123 CAR T.

\section{Name change}

\section{BioTime into Lineage Cell Therapeutics}

BioTime (CA, USA; www.biotimeinc.com) has announced it is launching a new corporate brand, including a change of its corporate name to Lineage Cell Therapeutics (CA, USA; www.lineagecell.com), effective 12 August 2019 [16]. In connection with the launch, the company's NYSE American ticker symbol will change to 'LCTX' and will be effective at the open of the market on 12 August 2019. The Company's new identity reflects its commitment to becoming an innovative, leading cell therapy company and highlights its extensive cell therapy platform. The Company also will be relocating its corporate headquarters from San Francisco Bay Area to CA, USA.

\section{Capital market \& finances \\ Century Therapeutics}

Century Therapeutics (PA, USA; www.centurytx.com), which was created by Versant Ventures (CA, USA; www.ve rsantventures.com) as part of the firm's core strategy to launch innovative biotech companies, emerged from stealth mode with US $\$ 250$ million in financing commitments from Bayer (Germany; https://leaps.bayer.com), Versant and FUJIFILM Cellular Dynamics (FCDI; WI, USA; https://fujifilmcdi.com) [17]. The proceeds will enable Century to advance multiple programs into the clinic for hematologic and solid malignancies.

Century's foundational technology is built on iPSCs that have unlimited self-renewing capacity. This enables multiple rounds of cellular engineering to produce master cell banks of modified cells that can be expanded and differentiated into immune effector cells to supply vast amounts of allogeneic, homogeneous therapeutic products. This platform differentiates Century from competitors that are developing cell therapies made from nonrenewable donor-derived cells.

Century was founded by Versant in 2018 and later that year formed a strategic partnership with FCDI to develop iPSC-derived immune effector cells for cancer. FCDI's vertically integrated iPSC platform has been optimized over the last 15 years to include the foremost reprograming technology that uses genome integration-free methods to generate GMP grade iPSC lines. Century also has exclusive access to FCDI's leading immune effector cell differentiation protocols and intellectual property to manufacture GMP-grade immune effector cells at commercial scale. Under the terms of the iPSC platform license agreement, FCDI will serve as the primary manufacturer of Century's cellular products. 


\section{Frequency Therapeutics}

Frequency Therapeutics (MA, USA; www.frequencytx.com), a clinical-stage biotechnology company focused on harnessing the body's innate biology to repair or reverse damage caused by a broad range of degenerative diseases, has closed US $\$ 62.0$ million Series C financing [18]. Frequency will use the proceeds to support the clinical development of FX-322, a regenerative therapeutic for the treatment of sensorineural hearing loss that is moving into a Phase IIa study, and to advance discovery programs in other therapeutic areas using its proprietary Progenitor Cell Activation platform. With the closing of the Series C, Frequency will have raised a total of US $\$ 147$ million in equity financings.

\section{Financial \& competing interests disclosure}

The author has received an honorarium from Future Science Group for the contribution of this work. The authors have no other relevant affiliations or financial involvement with any organization or entity with a financial interest in or financial conflict with the subject matter or materials discussed in the manuscript apart from those disclosed.

No writing assistance was utilized in the production of this manuscript.

\section{References}

1. ElevateBio. Massachusetts General Hospital and ElevateBio Announce a 10-Year alliance agreement to utilize ElevateBio BaseCamp to manufacture innovative cell and gene therapies (2019).

www.elevate.bio/press-release-blog/massachusetts-general-hospital-and-elevatebio-announce-a-10-year-alliance-agreement

2. FUJIFILM Cellular Dynamics. Fujifilm and Axcelead Drug Discovery Partners begin collaborating to advance drug discovery solutions using iPSCs (2019). https://fujifilmcdi.com/assets/Fujifilm_Axcelead.pdf

3. Epistem. Epistem licence Hubrecht Organoid Technology to expand preclinical toxicology service portfolio (2019) www.epistem.co.uk/resources/news/hubrecht-organoid-technology

4. Frequency Therapeutics. Astellas and frequency therapeutics enter into license agreement for FX-322, a regenerative treatment candidate for hearing loss (2019). www.frequencytx.com/news-events/news-events-press-release-07-17-2019.php

5. Humanigen. Humanigen secures exclusive worldwide license to gene-editing technology from Mayo Clinic to improve CAR-T (2019). www.humanigen.com/press/Humanigen-Secures-Exclusive-Worldwide-License-to-Gene-Editing-Technology-from-Mayo-Clinic-to-I mprove-CAR-T

6. Humanigen. Humanigen secures exclusive worldwide license for the prevention of GvHD through GM-CSF neutralization from the University of Zurich (2019). www.humanigen.com/press/Humanigen-Secures-Exclusive-Worldwide-License-for-the-Prevention-of-Gv HD-through-GM-CSF-Neutralization-from-the-University-of-Zurich

7. Anemocyte. Drones land in the cell and gene therapy space (2019). www.anemocyte.com/drones-land-in-the-cell-and-gene-therapy-space/

8. Kite Pharma. Kite announces plans to bolster industry-leading cell therapy manufacturing capabilities with new viral vector facility (2019). www.businesswire.com/news/home/20190716005214/en/Kite-Announces-Plans-Bolster-Industry-Leading-Cell-Therapy

9. StemoniX. StemoniX ${ }^{\circledR}$ opens new, state-of-the-art research \& development facility (2019). https://stemonix.com/new-state-of-the-art-research-development-facility/

10. BioTime. BioTime initiates dosing in Phase I/IIa clinical study of OpRegen for treatment of Dry-AMD utilizing orbit subretinal delivery system (2019). http://investor.biotimeinc.com/node/19856/pdf

11. Adaptimmune. Adaptimmune starts the SURPASS Clinical Trial with its first Next-Generation SPEAR T-cells targeting MAGE-A4 to enhance antitumor responses (2019). https://ir.adaptimmune.com/node/10236/pdf

12. Adaptimmune. Adaptimmune starts SPEARHEAD-1 trial with ADP-A2M4 SPEAR T-cells for patients with SynovialSarcoma or MRCLS (2019). https://ir.adaptimmune.com/node/10256/pdf

13. Elios Therapeutics. Elios Therapeutics announces positive top-line data from Phase IIb study evaluating TLPLDC, a personalized therapeutic cancer vaccine, in patients with high-risk melanoma (2019). www.eliostherapeutics.com/press/

14. Caladrius. Caladrius Biosciences receives advanced therapy medicinal product classification for CLBS12, its CD34+ cell therapy for critical limb ischemia (2019). www.caladrius.com/press-release/caladrius-biosciences-receives-advanced-therapy-medicinal-product-clas sification-for-clbs12-its-cd34-cell-therapy-for-critical-limb-ischemia/

15. Mustang Bio. Mustang Bio receives orphan drug designation for MB-102 (CD123 CAR T) for the treatment of acute myeloid leukemia (2019). http://ir.mustangbio.com/file/Index?KeyFile=398860454

16. BioTime. BioTime announces name change to lineage cell therapeutics (2019). http://investor.biotimeinc.com/node/19906/pdf

17. Century Therapeutics. Century Therapeutics launches with USD $250 \mathrm{M}$ financing for induced pluripotent stem cell (iPSC) allogeneic cell therapy platform (2019). www.centurytx.com/media/downloads/Century-Launch-PR_7.1.19_FINAL3.pdf

18. Frequency Therapeutics. Frequency Therapeutics completes $\$ 62$ million series C financing (2019). www.frequencytx.com/news-events/news-events-press-release-07-23-2019.php 